\title{
Studying space representation within a neuropsychological perspective
}

\author{
P. Zoccolotti • D. Spinelli • I. H. Robertson
}

Published online: 14 August 2010

(C) Springer-Verlag 2010

This special issue is in honour of Luigi (better known as Gino) Pizzamiglio, who devoted his long and productive scientific career to neuropsychological research and made significant contributions in areas ranging from phonology and language to space perception and human navigation. His approach is characterized by a highly innovative combination of experimental methods deriving from psychology, phonology, and cognitive neuroscience to study the disorders of individuals with circumscribed cerebral lesions and particular genetic profiles. With the advent of neuroimaging techniques, Gino began to use these methods to make further important contributions in the areas of neglect, human navigation, and representation of action. This all took place in parallel with his enduring interest in using experimental paradigms to study recovery and rehabilitation following brain damage.

After receiving his degree in Medicine, Gino specialized in Psychology at the Catholic University of Milan. He pursued his interests in phonology and language and collaborated with Professor John Black at Columbus University, in Ohio. In 1970, he moved to Rome. At this time,

P. Zoccolotti ( $\square)$

Department of Psychology, Sapienza University, Rome, Italy

e-mail: pierluigi.zoccolotti@uniroma1.it

P. Zoccolotti · D. Spinelli

Neuropsychology Unit, IRCCS Fondazione Santa Lucia,

Rome, Italy

\section{Spinelli}

Department of Education Sciences in Sport and Physical

Activity, University "Foro Italico", Rome, Italy

I. H. Robertson

Department of Psychology, Trinity College Dublin,

Dublin, Ireland when the first psychology degree programs were being established in Italy, Gino worked first at the Catholic University and then at Sapienza University. Here, during 40 years of intense activity, he made a fundamental contribution by promoting high-quality research in cognitive neuroscience and training successive generations of young scientists. In fact, over the years, many young neuroscientists and clinical neuropsychologists benefited from his suggestions, advice, and supervision.

One of his major contributions during this time was to establish the Neuropsychological Research Centre at the Fondazione Santa Lucia in Rome, which, thanks to his initiative, grew into a major research centre with a strong international profile. By locating the research centre in a large rehabilitation hospital, Gino created the conditions for the close integration of neuropsychological theory, clinical practice, and experimentation. Gino's work on the rehabilitation of spatial and language disorders is but one of many examples of his highly original and far-sighted approach.

The aim of this special issue is to present recent developments in research areas Gino has been particularly involved in, that is, space and attention. It includes papers written by several of his former students and co-workers, as well as by distinguished international researchers with similar research interests.

\section{Coding personal and extrapersonal space}

One topic concerns the mechanisms used in coding personal versus extrapersonal space and the possible modulating role of attention on these mechanisms.

The concept of reference frame is of fundamental importance in the field of spatial perception and memory. 
As Galati, Pelle, Berthoz, and Committeri summarize, neuroimaging studies show that many regions of the human brain are organized as structured spatial maps of locations or features of objects, the world, and our own body. Pizzamiglio and his collaborators have contributed to this line of research by carrying out studies on the cortical regions involved in body referencing using fMRI. These studies have been conducted in Rome and Paris (in a fruitful collaboration with Alain Berthoz's research group). Results show the involvement of parietal and frontal cortices in tasks requiring egocentric reference. During navigation in space, dynamic spatial updating of egocentric spatial maps compensates for body movements, but remembered spatial locations are also updated "offline" through the use of allocentric, environmental reference frames. A large body of studies are reviewed along these lines. New evidence is also provided indicating the parahippocampal and retrosplenial cortices as the neural substrates of environmental allocentric representations. The presence of objects whose position is stable over time (allowing for spatial learning) seems the critical factor in activating these areas.

Morrone, Cicchini, and Burr review data from their laboratory on the mechanisms for transforming coordinate systems to encode space, time, and motion. The human MT complex shows BOLD responses consistent with spatiotopic coding. This result complements psychophysical evidence that subthreshold motion signals are temporally integrated across saccades, but only when the two motion signals are in the same position in space. The fMRI results for MT contrast with other data in the literature showing retinotopic, rather than spatiotopic, responses. The authors propose that these divergent results may be due to the different allocation of attention in the critical experiments, raising the possibility that attention is essential for a spatiotopic organization to emerge. Recent data are also presented showing that mechanisms involved in timing the duration of events are anchored to robust external, realword (spatiotopic) coordinates.

Representing one's body requires the integration of input across several different modalities. These signals are normally consistent and complementary. Papeo, Longo, Feurra, and Haggard examine what happens when conflicting information must be integrated. They are particularly interested in clarifying the role of the right temporo-parietal junction (rTPJ), a region that processes multisensory bodyrelated information. Their experiment investigates whether the rTPJ is involved in resolving intersensory conflicts to maintain a coherent percept of the body or whether it simply detects the intersensory discrepancy.

With the paper by Aglioti and Pazzaglia, we move to a picture of space that is closer to the complex experience of the real word, i.e. the space in which we see and hear the sounds of others' actions. The authors review studies that focus on the representation of others' actions through audition, from monkey studies up to human action representation triggered by action sounds. Pizzamiglio has contributed to this issue with several studies indicating the existence of separate neural systems for sounds that can, or cannot, be translated into human actions. Reviewed data (as well as new data presented in the paper) indicate that somatotopy is an important representational rule for mapping action based on both visual observation and auditory perception. The authors discuss the importance of audiomotor mirroring for language and social interaction.

Driver, Blakenburg, Bestmann, and Ruff offer an original perspective for future research based on approaches integrating different techniques. The background is provided by data collected by the separate use of single approaches, in particular by observations on neurological patients and studies based on neuroimaging in normal subjects and patients. The view emerging from recent studies is that spatial attention is subserved by extended networks of interconnected brain areas. The use of concurrent techniques may allow measuring the effects of manipulations in a targeted area on functions in remote connected areas. The authors focus on the topic of attention and spatial cognition and review studies based on the concurrent use of TMS and fMRI, TMS combined with concurrent EEG, and fMRI and/or EEG in patients with focal lesions. Notably, the authors propose that this latter approach may also be useful to reveal the impact of interventions targeting a specific brain area on functions in remote interconnected areas.

\section{Environmental navigation}

The theme of environmental navigation represents one of Pizzamiglio's more recent research interests. His study of navigation in patients with neglect has been carried out primarily in collaboration with Cecilia Guariglia. In their review paper, Guariglia and Piccardi summarize the main points of this topic and also present some new data. These authors observe an important difference between patients with visuo-spatial (or perceptual) neglect and patients with pure representational neglect. Despite deficits in actual exploration, the former patients show some spared basic navigation skills. By contrast, impairments in developing, storing, and using cognitive maps affect some (but not all) patients with representational neglect. Guariglia and Piccardi also show the dissociation between mental representation of navigational space and mental representation of objects in patients with representational neglect.

The topic of navigation is further developed by Piccardi, Berthoz, Baulac, Denos, Dupont, Samson, and Guariglia. 
Following a suggestion by Pizzamiglio (reported in this issue by Guariglia and Piccardi) that spatial processes involved in navigation are dissociated from those involved in the processing of non-navigational information, they attempt to clarify whether there are two different memory systems, that is, one for processing memory for object locations and another for route and environment memory. Pertinent studies are summarized and new data, collected in the Unité d'Épilepsie of the Salpetrière Hospital, on patients with small circumscribed lesions in the middle temporal lobe (due to surgical treatment of intractable epilepsy) are presented. The authors assess the patients' ability to perform a spatial memory task in peripersonal or extrapersonal space and report a double dissociation, which supports the existence of two memory systems.

In previous studies of navigation abilities in neglect patients, Pizzamiglio and co-workers reported a double dissociation for deficits in using environmental landmarks and in using geometric information. This result is consistent with the proposal of different independent mechanisms for processing the position of visual cues and the shape of the environment. This theme has been the main focus of research by Elizabeth Spelke and co-workers. Although Pizzamiglio never collaborated with Spelke, he was impressed by Spelke's work and incorporated several related concepts into his studies.

In the present issue, Lee and Spelke review evidence supporting the hypothesis of two independent cognitive processes by which spatial properties of the environment are analysed for the purpose of navigation. According to their proposal, one automatic process uses the shape of surfaces, and distances and the direction of surfaces specify the navigator's position. A second process is based on objects and 2D patterns. This process is attention dependent and uses objects and surface features as direct markers of location, but not as relative position cues for reorientation. The authors discuss developmental changes in terms of the emergence of new navigation processes that may depend on the capacity for symbolic representation, a uniquely human system of spatial representation constructed from independent parallel system of navigation and object recognition.

A final contribution to the topic of navigation is provided by a former student of Pizzamiglio, Giuseppe Iaria, who left Italy and now works in Canada. In a recent study, Iaria and co-workers reported the case of a woman with a selective impairment in orienting in a familiar environment. The absence of cerebral injuries or malformations and the presence of intact cognitive skills led them to propose a new disorder: developmental topographical disorientation. In the present study, Iaria and Barton move beyond the single case approach and report results from a new database of 120 patients. The inability to form a cognitive map is the most consistent defect in this population. Individuals with this impairment get lost almost daily in their most familiar surroundings, such as their home and the neighbourhood where they have lived for several years. Future research is urged to clarify the structural, functional, and genetic aspects of the disorder.

\section{Unilateral spatial neglect}

In the last 25 years, unilateral spatial neglect (or neglect) has been one of the Pizzamiglio's most productive areas of research. In the present issue, four papers refer to this topic.

In studies on neglect, key questions are attentional dysfunction and its underlying neural substrates. As Shomstein, Lee, and Behrmann summarize, recent studies have fragmented the parietal cortex into subcomponents and the attentional disorder into differing subprofiles. The authors present an experimental contribution aimed at distinguishing brain lesions associated with goal-driven attentional control deficits and stimulus-driven attentional orienting deficits in a group of patients with neglect. Two distinct anatomical sites are critical, one mediating topdown attentional orienting (superior parietal lobe) and the other mediating bottom-up capture of attention (temporoparietal junction). The patients were assigned to different groups according to their behavioural profiles, and their lesion overlap was studied.

Spatial neglect most frequently results from cortical to subcortical lesions in the territory of the middle cerebral artery area. However, as Tomaiuolo, Doricchi, and coworkers summarize, spatial neglect can also result from lesions in the posterior cerebral artery (PCA) area. The authors present a study of two patients with PCA lesions selectively affecting the splenium of the corpus callosum, the adjacent right primary visual cortex, and the optic radiations. The authors show that neglect is selectively restricted to the contralesional visual space. Motor and personal-body neglect are absent, as well as hemispatial limb akinesia, contralesional tactile extinction, and contralesional tactile anomia. This pattern of clinical dissociation has never been described before; in all previously reported patients with PCA lesions, the callosal damage always spared the splenium or entirely encompassed the corpus callosum.

Neglect dyslexia (ND) has been investigated in the neuropsychological literature for over a century. Vallar, Burani, and Arduino summarize the history of ND, which dates back to Arnold Pick's early observations in 1898. The deficit has been investigated either as one of the aspects of spatial neglect or as it relates to the reading system. In the latter case, ND is classified as one of the forms of peripheral dyslexia. A comprehensive review of all cases 
reported in the literature is presented with a special emphasis on the analysis of reading errors and the presence of preserved lexical and morphological processing for misread stimuli. Particular attention is also given to the neuroanatomical correlates of ND and how these interact with different levels of processing of the letter string. Along these lines, a neuro-functional model is presented that aims to integrate the analysis of linguistic stimuli with the relative contribution of the two cerebral hemispheres.

The special issue ends with the paper by Cappa and Perani. The authors note Pizzamiglio's long-standing interest in the neural mechanisms responsible for spontaneous and rehabilitation-induced recovery after brain lesions, particularly in neglect patients. They recount Pizzamiglio's enthusiasm for the new neuroimaging techniques and his pioneering work in collaboration with the Milan group in the late 1980s. The reviewed neuroimaging studies of neglect patients indicate that multiple structures, both ipsilateral and contralateral to the lesion, are involved in recovery and that rehabilitation influences the pattern of brain reorganization. Notably, changes in activation take place within dedicated neural networks, underscoring the limits of the plasticity of the human adult brain. In considering the direction of future research, Cappa and Perani point out the need to continue Pizzamiglio's pioneering work in the area of recovery. They encourage more in vivo studies of anatomical and functional connectivity to address this most crucial question, which constitutes the raison d'etre of cognitive neuroscience.

\section{Concluding remarks}

Gino Pizzamiglio has made an invaluable contribution to the enormous progress in understanding the representation of space that has occurred over the past three decades. This testifies to his intellectual curiosity, scientific leadership, and scholarly creativity. He was a pioneer in this area and also in studying how people recover when these and other neuropsychological systems are damaged. His work on rehabilitation was far ahead of its time, and he was one of the first to apply neuroimaging techniques to study recovery and rehabilitation mechanisms. We believe that this special issue is a fitting testimony to an outstanding scientific career. 